\title{
Enfermedad de depósito glomerular: a propósito de un caso de glomerulonefritis fibrilar
}

\author{
Jorge de Jesús Cantillo1,2, Rocío del Pilar López ${ }^{3,4}$, Rafael Enrique Andrade \\ 1 Servicio de Nefrología y Diálisis, Hospital El Tunal, E.S.E., Bogotá, D.C., Colombia \\ 2 Servicio de Terapia Renal, Hospital Universitario Clínica San Rafael, Bogotá, D.C., Colombia \\ ${ }^{3}$ Departamento de Patología y Laboratorios, Hospital Universitario Fundación Santa Fe de Bogotá, Bogotá, \\ D.C., Colombia \\ 4 Facultad de Medicina, Universidad de los Andes, Bogotá, D.C., Colombia \\ 5 Facultad de Medicina, Universidad Nacional de Colombia, Bogotá, D.C., Colombia
}

En el diagnóstico rutinario por biopsia renal encontramos enfermedades glomerulares que tienen depósitos estructurales fibrilares en la matriz extracelular del glomérulo, visibles por microscopía electrónica. La amiloidosis es la glomerulopatía más común con depósitos de estas características ultraestructurales. Sin embargo, hay otras glomerulopatías con depósitos similares, negativas con rojo Congo, pero positivas para inmunoglobulinas.

La glomerulonefritis fibrilar es una entidad diagnóstica ampliamente reconocida e identificada en, aproximadamente, $0,5 \%$ a $1 \%$ de las biopsias de riñones nativos. Suele manifestarse por una insuficiencia renal progresiva con síndrome nefrótico-nefrítico e hipertensión arterial sistémica, no tiene tratamiento claramente establecido y su pronóstico es malo.

Se describen las características clínicas e histológicas del primer caso de glomerulonefritis fibrilar idiopática informado en Colombia.

Palabras clave: glomerulonefritis, síndrome nefrótico, inmunoglobulinas, insuficiencia renal, informes de casos.

\section{Glomerular deposition disease, regarding a case of fibrillary glomerulonephritis}

In routine diagnosis for renal biopsy, glomerular diseases are encountered that, viewed by electron microscopy, reveal deposits with a fibrillary structure in the extracellular matrix of the glomeruli. Amyloidosis is the most common glomerulopathy that shows deposits with an ultrastructural fibrillary structure. However, other glomerulopathies have deposits with an ultrastructural fibrillary structure and which are negative for Congo-red stain, but positive for immunoglobulins.

Fibrillary glomerulonephritis is a well-characterized disease, and clearly identified in approximately $0.5 \%$ to $1 \%$ of biopsies of native kidneys. These cases usually manifest themselves as severe renal insufficiencies with nephrotic-range proteinuria. No treatment regimes have been clearly established and the prognosis is poor. Herein, the clinical and histological characteristics are described for the first case of idiopathic fibrillary glomerulonephritis reported in Colombia.

Key words: glomerulonephritis, nephrotic syndrome, immunoglobulins, renal insufficiency, case reports.

Esta glomerulopatía fue descrita por vez primera en 1977, cuando Rosenmann y Eliaakim observaron, en el glomérulo, un material fibrilar

\footnotetext{
Correspondencia:

Jorge de Jesús Cantillo, Calle 114 A № 56-34, apartamento 601, Bogotá, D.C., Colombia

Teléfonos: (571) 714 4372, 560 2009, 5603284 y 5602464

md_cantillo@yahoo.com, md_cantillo@hotmail.com
}

Recibido:15/04/09; aceptado:02/07/09 similar al amiloide pero que no compartía su afinidad por el rojo Congo. Más tarde, en 1983, Duffy et al. describieron ocho pacientes con hipertensión, hematuria y proteinuria, en cuyas biopsias se observaron depósitos de fibrillas dispuestas al azar en el mesangio y transmembranoso; utilizaron el término fibrilar para designar la ultraestructura de dichos depósitos. 
Sin embargo, el nombre de glomerulonefritis fibrilar fue acuñado en 1987 por Alpers et al., quienes describieron en siete casos la presencia de depósitos extracelulares de fibrillas orientadas al azar y de un diámetro superior al del amiloide (10 a $20 \mathrm{~nm}$ ), las cuales contenían inmunoglobulinas y complemento. Casi simultáneamente, en 1980, Schwartz y Lewis describieron otra glomerulopatía con estructuras microtubulares de mayor tamaño (aproximadamente, $27 \mathrm{~nm}$ ) y depósitos inmunes monoclonales IgG kappa dispuestos en bandas paralelas, hallazgos que definen la glomerulopatía inmunotactoide.

Actualmente, se sigue presentando confusión en la nomenclatura de estas glomerulopatías. Pese a que varios autores divulgaron durante años el nombre de "glomerulonefritis fibrilar inmunotactoide", considerando que ambas glomerulopatías formarían una misma entidad clínico-patológica sin criterios clínicos y patológicos suficientes como para justificar una subclasificación, son cada vez más numerosos los autores que opinan que estas entidades deben separarse en virtud, no sólo de sus diferencias ultraestructurales, sino del tipo de depósito inmune, y a las cada vez más importantes implicaciones clínicas y renales que se documentan en las series y casos publicados (1).

El objetivo de este trabajo es informar el primer caso de esta glomerulopatía en la literatura nacional.

\section{Presentación del caso}

Se trata de un hombre de 51 años de edad, remitido a consulta de nefrología por proteinuria en rango nefrótico $(12,7 \mathrm{~g})$ y elevación de la creatinina sérica $(1,65 \mathrm{mg} / \mathrm{dl})$. Refería edema de miembros inferiores y orinas espumosas.

Entre sus antecedentes presentaba obesidad e hipertensión arterial sistémica diagnosticada siete años antes y manejada con nifedipina de liberación lenta, enalapril y clonidina.

En el examen físico, se encontraba en buenas condiciones generales, con temperatura de $36^{\circ} \mathrm{C}$, tensión arterial de 150/100 mm Hg, frecuencia respiratoria de 15 por minuto, frecuencia cardiaca de 70 por minuto y peso de $84 \mathrm{~kg}$. Presentaba edema grado II de miembros inferiores. El resto del examen no mostró hallazgos positivos.

Los exámenes paraclínicos se resumen en el cuadro 1. La ecografía renal reveló incremento de la ecogenicidad cortical, con preservación de la relación córtico-medular, y tamaño y contornos renales normales.

Como diagnósticos presuntivos se consideraron la enfermedad glomerular, el síndrome nefróticonefrítico, la hipertensión arterial sistémica no controlada, la obesidad y la enfermedad renal crónica en estadio 3 . Se reajustó la terapia antihipertensiva proporcionando mayor efecto antiproteinúrico, dieta hipoproteica con $0,8 \mathrm{~g}$ de proteínas por $\mathrm{kg}$ de peso al día (un gramo más de proteína por gramo de proteinuria), dieta hiposódica e hipolipidemiantes orales, y se ordenaron exámenes para enfermedad glomerular (cuadro 1).

Considerando los hallazgos descritos, se practicó biopsia renal percutánea, cuyo examen por microscopía de luz mostró: número de glomérulos, 39 (11 con esclerosis global y 1 con esclerosis segmentaria); proliferación celular endocapilar/del mesangio (+++); engrosamiento difuso de las membranas basales capilares $(++)$; dobles contornos $(++)$, ensanchamiento del mesangio $(++)$ con áreas que contenían un material rosado amorfo, negativo para rojo Congo; fibrosis túbulo-intersticial $(++)(60 \%)$; nefritis intersticial $(++)(30 \%)$; mononucleares polimorfonucleares y plasmocitos; atrofia tubular $(++) \quad(50 \%)$; engrosamiento de membranas basales tubulares $(++)$; reacción negativa para amiloide con rojo Congo; esclerosis arterial (de la subíntima, +; de la media, +); esclerosis arteriolar (de la subíntima, +); hialinosis (++).

La inmunofluorescencia reveló: IgG (++) en la membrana basal capilar y membrana basal tubular; IgA, trazas o negativa en la membrana basal capilar; cilindros $(++)$; lgM, trazas en la membrana basal tubular; C3 (++) en la membrana basal capilar con patrón granular. No hubo otros hallazgos positivos. 
Cuadro 1. Resultados de laboratorio clínico de ingreso.

\begin{tabular}{ll}
\hline Creatinina & $1,68 \mathrm{mg} / \mathrm{dl}(0,7-1,3)$ \\
BUN & $29,6 \mathrm{mg} / \mathrm{dl}(7-20)$ \\
Potasio & $4,8 \mathrm{mmol} / \mathrm{L}(3,5-5,1)$ \\
Sodio & $137 \mathrm{~mm} / \mathrm{L}(136-145)$ \\
Ácido úrico & $8,4 \mathrm{mg} / \mathrm{dl}(2,4-7)$ \\
ALAT & $26 \mathrm{U} / \mathrm{L}(0-55)$ \\
ASAT & $29 \mathrm{U} / \mathrm{L}(5-34)$ \\
Colesterol total & $243 \mathrm{mg} / \mathrm{dl}(0-200)$ \\
Glucemia & $121 \mathrm{mg} / \mathrm{dl}(70-109)$ \\
HDL & $41 \mathrm{mg} / \mathrm{dl}(35-65)$ \\
LDL & $147 \mathrm{mg} / \mathrm{dl}(0-190)$ \\
Proteínas totales & $6,11 \mathrm{~g} / \mathrm{dl}$ \\
Albúmina & $2,9 \mathrm{~g} / \mathrm{dl}(3,5-5)$ \\
Triglicéridos & $678 \mathrm{mg} / \mathrm{dl}(0-149)$ \\
Cuadro hemático & Hemoglobina: $16 \mathrm{~g} / \mathrm{dl}(12,2-18,1)$ \\
& Hematocrito: $46 \%(37,7-53,7)$ \\
& Plaquetas: $328.000 \times \mathrm{mm}{ }^{3}(142.000-$ \\
& $424.000)$ \\
Uroanálisis & Leucocitos: 11.500 \\
& Proteínas: $300 \mathrm{mg} / \mathrm{dl}$ \\
ANA & Hematíes: $5-6 \mathrm{por}$ campo \\
C-ANCA & Negativos \\
p-ANCA & Negativo \\
C3 & Negativo \\
C4 & $2,11 \mathrm{mg} / \mathrm{dl}(82-193)$ \\
Anti-HVC & $0,37 \mathrm{mg} / \mathrm{dl}(15-57)$ \\
Anti-VIH & Negativo \\
Anti-HBsAg & Negativo \\
Proteinuria orina de & $2,1 \mathrm{mUl} / \mathrm{ml}$ \\
24 horas & $12,7 \mathrm{~g}$ \\
Crioinmunoglobulinas \\
séricas & Negativas \\
Electroforesis de & \\
proteínas & Negativa \\
& \\
\hline &
\end{tabular}

En la microscopía electrónica se encontró engrosamiento difuso de la membrana basal capilar con presencia de dobles contornos e interposición del mesangio. No se encontraron depósitos con características de complejos inmunes en ninguna localización. La matriz del mesangio estaba expandida. Había grandes masas de depósitos organizados de aspecto fibrilar, dispuestos al azar, de localización, principalmente, en el mesangio e intramembranosa, que medían menos de $25 \mathrm{~nm}$.

Por anatomopatología se diagnosticó glomerulonefritis fibrilar y nefritis túbulo-intersticial crónica (figuras 1 a 4).

Ante la presencia de un paciente con síndrome nefrótico persistente con intensa proteinuria, y a pesar de los hallazgos de nefritis túbulointersticial crónica y de los antecedentes de casos de glomerulonefritis fibrilar sin enfermedad sistémica subyacente o asociada con respuesta insatisfactoria a corticoterapia e inmunosupresión, se decidió hacer un primer intento con prednisolona a $0,5 \mathrm{mg} / \mathrm{kg}$ diarios más ciclofosfamida oral a $2 \mathrm{mg} / \mathrm{kg}$ diarios. La respuesta fue nula y un segundo intento con micofenolato de mofetilo también fracasó. Por lo tanto, se tomó la decisión de intensificar el control de los factores de progresión de la enfermedad renal.

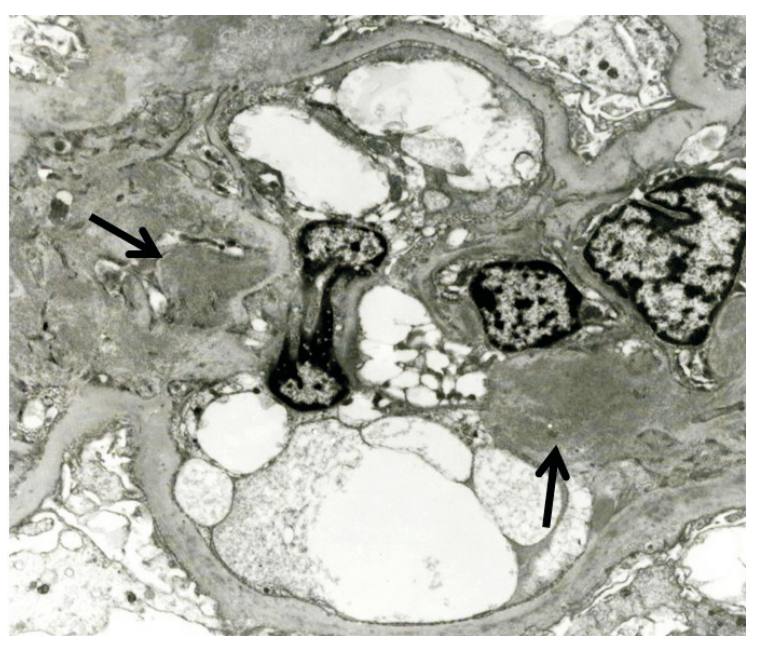

Figura 1. Depósitos electrón-densos organizados de características fibrilares en localización en el mesangio. Aumento original: 5.000X por microscopía electrónica

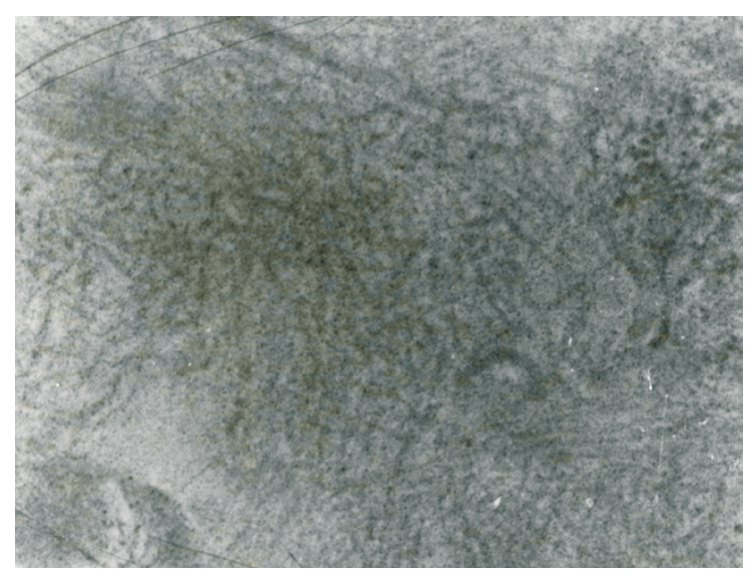

Figura 2. Depósitos electrón-densos organizados, de características fibrilares en localización en el mesangio. Aumento original: $30.000 X$ por microscopía electrónica 


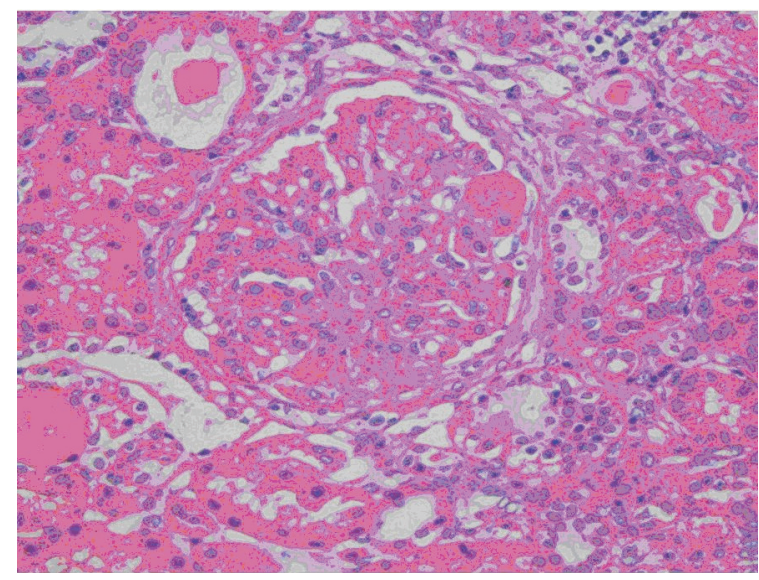

Figura 3. Expansión del mesangio y engrosamiento difuso de membranas basales capilares con obliteración de la luz. Presencia de material hialino. Hematoxilina eosina, 40X.

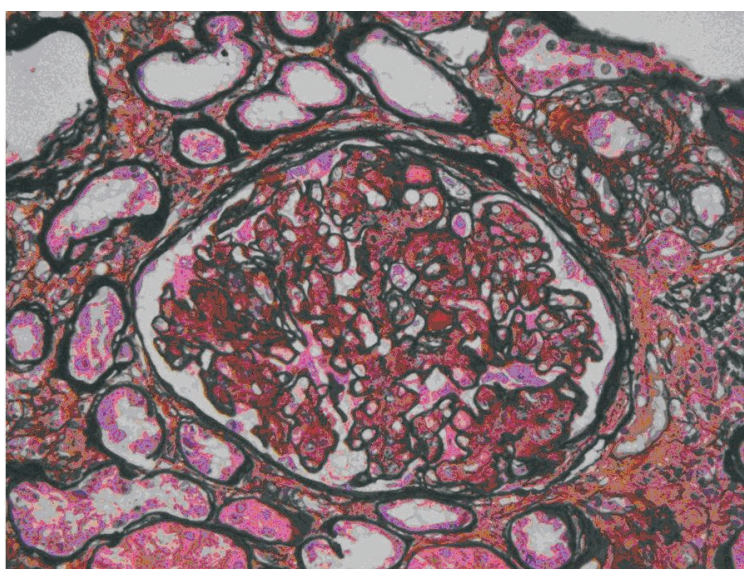

Figura 4. Engrosamiento difuso de membranas. Expansión de la matriz por material negativo con la plata. Plata metenamina, 20X.

Durante el seguimiento, su evolución clínica se caracterizó por dificultad en el control de las cifras de tensión arterial, persistencia de la obesidad, proteinurias en rango nefrótico persistentemente elevadas, difícil control de la dislipidemia y deterioro lento pero progresivo de la función renal. Después de tres años de seguimiento, ingresó al servicio de urgencias por uremia e hipervolemia, con creatinemia de $12 \mathrm{mg} / \mathrm{dl}$ y BUN de $96 \mathrm{mg} / \mathrm{dl}$, por lo que se inició hemodiálisis urgente. Dos meses después fue transferido a un programa de diálisis peritoneal crónica, en el cual permanece actualmente.

\section{Discusión}

El presente caso clínico de glomerulonefritis fibrilar es el primero que se informa en Colombia.

Esta glomerulonefritis forma parte de las denominadas enfermedades renales de depósito no amiloideas, entidades poco frecuentes y de reciente descripción en la literatura $(1,2)$. Hace parte de la familia de las enfermedades de depósito glomerular con estructuras fibrilares, entre las que también se encuentran la glomerulopatía amiloidea y la crioglobulinemia, cuyas ultraestructuras se caracterizan por la presencia de microfibrillas distribuidas al azar (diámetro: 15-30 nm) y constituidas, según la inmunofluorescencia, por IgG oligoclonal, negativa con rojo Congo y asociada a baja incidencia de enfermedades sistémicas subyacentes (3-5).

La glomerulonefritis fibrilar es una causa de enfermedad glomerular cada vez más reconocida y el diagnóstico ha sido identificado en, aproximadamente, $1 \%$ de las biopsias renales en adultos (riñones nativos) en, al menos, tres grandes series de biopsias informadas hasta la fecha (6-8). Puede presentarse a cualquier edad; sin embargo, usualmente es una enfermedad de adultos, más frecuente a los 50 años (nuestro caso se presentó a los 51 años). Los enfermos suelen ser de raza blanca, con un ligero predominio femenino (9).

Clínicamente, nos enfrentamos a un paciente en la edad media de la vida, con antecedentes de hipertensión arterial sistémica diagnosticada siete años antes, con signos de enfermedad glomerular de presentación nefrótica-nefrítica, deterioro de la función renal (enfermedad renal crónica en estadio 3) y ecografía renal normal. La mayoría de los veces, la glomerulonefritis fibrilar se acompaña de proteinuria elevada, por lo general, en rango nefrótico $(50 \%)$, junto con hematuria e hipertensión $(60 \%$ y $77 \%$, respectivamente, en algunas series) $(1,5,9)$.

La presencia de proliferación endocapilar y proliferación del mesangio, con su consiguiente expansión y engrosamiento difusos con aspecto 
de doble contorno de membranas basales capilares y la acentuación de la lóbulos, determinan la presencia de un patrón membranoproliferativo en el estudio de microscopía óptica.

Este tipo de patrón puede observarse en $73 \%$ de los casos de glomerulonefritis fibrilar y no es diagnóstico de esta entidad. Es menos frecuente encontrar un patrón proliferativo en el mesangio(13\%) o endocapilar difuso. Algunos casos pueden, incluso, parecerse a la nefropatía diabética, con grandes expansiones nodulares de la matriz del mesangio $y$, en algunas ocasiones, proyecciones en forma de espinas de la membrana basal glomerular, formando un patrón de tipo membranoso (15\%), que reflejan, al igual que la nefropatía membranosa, una reacción de la membrana basal glomerular ante los depósitos fibrilares de localización subepitelial.

La proliferación extracapilar con formación de semilunas celulares o fibrocelulares, se identificó en un tercio de los casos de una serie $(2-5,9,10)$. En nuestro caso, se observó ensanchamiento del mesangio $(++)$ con áreas que contenían un material rosado amorfo, negativo con tinción de plata y negativo para amiloide con rojo Congo, que corresponde a los depósitos mencionados en la ultraestructura. En muchos casos, un patrón de infiltración de estructuras glomerulares por un material acelular amorfo es la manifestación más llamativa, no acompañada por proliferación celular prominente (5). Este material infiltra regiones del mesangio (nuestro caso) y paredes capilares periféricas (5).

De la misma forma que en nuestro caso, más de $95 \%$ de los casos de las series publicadas muestran $\lg G$ y $C 3$, IgM en $60 \%$ e $\lg A$ en $30 \%$ (positividad más débil) (11). A diferencia de nuestro caso, en la mayoría de los pacientes están presentes ambas cadenas livianas, kappa y lambda $(6,7)$. Sólo en $10 \%$ de los casos informados en la literatura se describen depósitos monoclonales, por lo general, IgG kappa.

Iskandar et al. (7), en una amplia serie publicada en 1992 sobre glomerulonefritis fibrilar (excluyendo los casos de glomerulopatía inmunotactoide), en el estudio de subclases, observaron que la mayoría de los depósitos estabanconformadospor lgG4 policlonal enforma dominante o exclusiva, planteando la hipótesis de la necesidad de una relativa homogeneidad de la inmuinoglobulina en los depósitos inmunes para favorecer la disposición fibrilar. En la glomerulopatía inmunotactoide, también los depósitos más frecuentes han sido de lgG y C3. Sin embargo, los depósitos de IgG4 policlonal suelen ser menos frecuentes $y$, por el contrario, el carácter monoclonal de los depósitos parece ser la característica dominante, a diferencia de lo que ocurre en la glomerulonefritis fibrilar. Por lo tanto, los hallazgos inmunohistoquímicos indican que los depósitos de microfibrillas estarían conformados por inmunoglobulinas y complemento, como lo demostraron Yang et al. (12) mediante inmunomicroscopía electrónica, quienes sugirieron que las fibrillas representarían componentes proteicos polimerizados (1-5,9).

El estudio de microscopía electrónica se hace imprescindible para el diagnóstico anatomopatológico. Nuestro caso mostró la presencia de grandes masas de depósitos organizados fibrilares, dispuestos al azar, de localización principalmente mesangial e intramembranosos, que miden menos de $25 \mathrm{~nm}$. Estos hallazgos confirman el diagnóstico de glomerulonefritis fibrilar, al demostrar la presencia de fibrillas alineadas de forma aleatoria que parecen fibrillas de amiloide, pero cuyo diámetro es mayor.

No obstante, no es posible distinguir esta entidad de la amiloidosis sólo por el diámetro de las fibrillas, según la experiencia de Fogo. Los diámetros de las fibrillas de algunas series han oscilado entre 20 y $22 \mathrm{~nm}$, con valores entre 13 y $39 \mathrm{~nm}$, aspecto que contrasta con las fibrillas de amiloide, que miden en promedio $10 \mathrm{~nm}$. Sin embargo, en la serie de glomerulonefritis fibrilar informada por Fogo, se encontró cierta superposición, con un diámetro medio de las fibrillas de la glomerulonefritis fibrilar de $14 \mathrm{~nm}$ (intervalo: 10,4 a 18,4 nm).

Por estas razones, resulta esencial demostrar la falta de tinción con rojo Congo y la inmunofluorescencia típica, según se comentó 
antes, como criterios diagnósticos. La microscopía electrónica muestra fibrillas en todos los compartimentos glomerulares, incluidos el mesangio, el subepitelio y el subendotelio y, en algunos casos, se han observado depósitos densos adicionales sin una composición fibrilar definida. Además, pueden encontrarse raros depósitos fibrilares en la membrana basal tubular (9).

Mientras la glomerulonefritis fibrilar es una glomerulopatía con estructurafundamentalmente fibrilar, caracterizadaporlaexistenciadepequeñas fibrillas de aspecto sólido en los aumentos de rutina, en la variante "inmunotactoide" (también de estructura fibrilar), las microfibrillas son más grandes (30 a $60 \mathrm{~nm}$ ), suelen tener luces visibles (un centro hueco) conformando microtúbulos, característicamente se agrupan conformando bandas paralelas, que pueden adoptar un patrón en "pilas de madera", y predominan los depósitos en el subendotelio y en el mesangio. Además, algunos casos pueden tener depósitos en el subendotelio o intramembranosos $(4,13)$. Por definición, la tinción con rojo Congo es negativa para amiloide, al igual que en la glomerulonefritis inmunotactoide (13).

Los autores partidarios de diferenciar ambas entidades se apoyan, no sólo en las diferencias ultraestructurales, sino en la trascendencia clínica que ello conllevaría (1). De un lado, Alpers (14) propone considerar la glomerulopatía "inmunotactoide" como una entidad distinta de la glomerulonefritis fibrilar por su fuerte asociación a procesos linfoproliferativos y disproteinemias, a diferencia de lo que ocurre con ésta última.

En 1993, Fogo et al. (6), en una serie de 26 casos de glomerulonefritis fibrilar y 6 casos de glomerulopatía inmunotactoide, observaron diferencias clínicas notables. En este trabajo se observó que los pacientes con glomerulonefritis fibrilar presentaban un peor pronóstico renal, describiéndose en sólo un caso mieloma múltiple. Por el contrario, en 4 de los 6 pacientes con glomerulopatía inmunotactoide se demostró asociación con paraproteinemia, presentaron una edad media más avanzada, y se comprobó a largo plazo un mejor pronóstico renal.
En otra gran serie reciente, presentada por Bridoux et al. (15), se incluyeron 14 casos de glomerulopatía inmunotactoide, en 10 de los cuales se identificaron procesos neoplásicos hematológicos. Por otra parte, en ninguno de los 9 casos de glomerulonefritis fibrilar que se describieron en este trabajo, se observó asociación con procesos linfoproliferativos o discrasias sanguíneas. Aunque estos autores no encontraron diferencias significativas entre las dos entidades en cuanto a supervivencia renal, sí se pudo comprobar que la administración de quimioterapia permitió la remisión del síndrome nefrótico en los 10 pacientes con glomerulopatía inmunotactoide y neoplasia hematológica (1).

Los autores partidarios de considerar la glomerulonefritis fibrilar e inmunotactoide como una misma entidad clínico-patológica (11), se apoyan en que las diferencias ultraestructurales no corresponden con las diferencias clínicas validadas estadísticamente y en la falta de consenso real en diferentes estudios sobre la existencia de una mayor asociación de glomerulonefritis fibrilar con enfermedades hematológicas. Otros autores prefieren utilizar el nombre de "glomerulonefritis fibrilar inmunotactoide", en virtud de la frecuente similitud ultraestructural entre ambas variantes y la inconstante asociación entre carácter monoclonal y tamaño de la fibrilla (16). En opinión de Brady et al. (17), el término de glomerulonefritis fibrilar inmunotactoide definiría mejor esta enfermedad por depósito glomerular, hasta que nuevos estudios arrojen más evidencias sobre la necesidad de diferenciar estas dos variantes (1).

Aunque se han descrito numerosas asociaciones con otras enfermedades, como procesos linfoproliferativos $(14,15)$, mieloma $(6,18)$, síndrome de Sjögren (6), vasculitis leucocitoclástica (19) y crioglobulinemia (20), la mayoría de los casos son idiopáticos (21). A favor de la patogenia múltiple de estas entidades, merece destacarse la comunicación de recientes casos de glomerulonefritis fibrilar y glomerulopatía inmunotactoide en pacientes con infección por el virus de la hepatitis C $(21,22)$; en uno de los cuales se informó normalización 
de la función renal tras completar la terapia con interferón (22).

Recientemente, se han comunicado también dos casos de coinfección con el virus de la inmunodeficienciahumanay el virus de la hepatitis C, asociados a estas glomerulopatías (23). El tratamiento médico actual de la glomerulonefritis fibrilar e inmunotactoide en los casos en los que no se describe enfermedad sistémica subyacente o asociada, es insatisfactorio con corticoterapia e inmunosupresión $(15,18)$. Además, se ha ensayado el trasplante renal, observándose en la mayoría de los casos recurrencia de la enfermedad, aunque el deterioro de la función renal evolucionó invariablemente más lentamente, por lo que se considera una opción atractiva en pacientes con enfermedad renal terminal (2).

\section{Agradecimientos}

A Jaime Muñoz Carreño por su contribución en la edición final de este trabajo y a Adriana Flórez y Paula Esquinas por la elaboración de las microfotografías.

\section{Conflicto de intereses}

No existe conflicto de intereses entre los autores, las instituciones y el contenido del presente trabajo.

\section{Financiación}

Declaramos que para la elaboración de este manuscrito no se ha contado con ninguna fuente de financiación.

\section{Referencias}

1. Vera FJ, Molina M, Hernández MA, García S. Glomerulonefritis fibrilar e inmunotactoide: descripción de un caso y revisión de la literatura. An Med Interna. 2005;22:35-8.

2. Pronovost PH, Brady HR, Gunning ME, Espinoza O, Rennke HG. Clinical features, predictors of disease progression and results of renal transplantation in fibrillary/immunotactoid glomerulopathy. Nephrol Dial Transplant. 1996;11:837-42.

3. Joh K. Pathology of glomerular deposition diseases and fibrillary glomerulopathies associated with paraproteinemia and haematopoietic disorder. Nephrology. 2007;12:S21-4.
4. Joh K. Pathology of glomerular deposition diseases. Pathol Int. 2007;57:551-65.

5. Alpers CE, Kowalewska J. Fibrillary glomerulonephritis and immunotactoid glomerulopathy. J Am Soc Nephrol. 2008;19:34-7.

6. Fogo A, Quereshi N, Hom RG. Morphological and clinical features of fibrillary glomerulonephritis versus immunotactoid glomerulopathy. Am J Kidney Dis. 1993;22:367-77.

7. Iskandar SS, Falk RJ, Jennette JC. Clinical and pathologic features of fibrillary glomerulonephritis. Kidney Int. 1992;42:1401-7.

8. Rosenstock JL, Markowitz GS, Valeri AM, Sacchi G, Appel GB, D'Agati VD. Fibrillary and immunotactoid glomerulonephritis: distinct entities with different clinical and pathologic features. Kidney Int. 2003;63:145061.

9. Fogo $\mathbf{A B}$, Kashgarian $\mathbf{M}$. Glomerulonefritis fibrilar. En: Fogo $A B$, Kashgarian $M$, editores. Atlas diagnóstico de patología renal. Madrid: Elsevier S.A.; 2006. p. 84-92.

10. Müller-Höcker J, Weiss M, Sitter Th, Samtleben W. Fibrillary glomerulonephritis mimicking membranous nephropathy- A diagnostic pitfall. Pathol Res Pract. 2009;205:265-71.

11. Korbet SM, Schwartz MM, Lewis EJ. The fibrillary glomerulopathies. Am J Kidney Dis. 1994;23:751-65.

12. Yang GC, Nieto R, Stachura I, Gallo GR. Ultrastructural immunohistochemical localization of polyclonal lgG, C3 and amyloid $\mathrm{P}$ component on the Congo red-negative amyloid-like fibrils of fibrillary glomerulopathy. Am J Pathol. 1992;141:409-19.

13. Fogo $\mathbf{A B}$, Kashgarian $\mathbf{M}$. Glomerulopatía inmunotactoide. En: Fogo $A B$, Kashgarian $M$. editores. Atlas diagnóstico de patología renal. Madrid; Elsevier S.A., 2006. p. 92-6.

14. Alpers CE. Immunotactoid(microtubular)glomerulopathy: an entity distict from fibrillary glomerulonephritis? Am J Kidney Dis. 1992;19:185-91.

15. Bridoux F, Hugue V, Coldefy O, Goujon JM, Bauwens $\mathbf{M}$, Sechet $\mathbf{A}$, et al. Fibrillary glomerulonephritis and immunotactoid (microtubular) glomerulopathy are associated with distinct immunologic features. Kidney Int. 2002;62:1764-75.

16. García I, Valenzuela M. Glomerulopatías con depósitos fibrilares. Rev Esp Patol. 2002;35:167-76.

17. Brady HR. Fibrillary glomerulopathy. Kidney Int. 1998:53:1421-9.

18. Vigil A, Oliet A, Gallar P, Ortega $\mathbf{O}$, Rodríguez I, Picazo L, et al. Rapidly progressive immunotactoid glomerulonephritis and multiple mieloma. Nephron. 1998;79:238-40. 
19. Orfila C, Meeus F, Bernadet P, Lepert JC, Sue JM. Immunotactoid glomerulopathy and cutaneus vasculitis. Am J Nephrol. 1991;11:67-72.

20. King J, Culppeper M, Corey R, Tucker A, Lajoie G, Howell D. Glomerulopathies with fibrillary deposits. Ultraestruct Pathol. 2000;24:5-21.

21. Markowitz G, Cheng J, Colvin R, Trebbin W, D'Agati V. Hepatitis $\mathbf{C}$ infection is associated with fibrillary glomerulonephritis and immunotactoid glomerulopathy. J Am Soc Nephrol. 1998;9:2244-52.
22. Coroneos E, Truong L, Oliveo J. Fibrillary glomerulonephritis associated with hepatitis C viral infection. Am J Kidney Dis. 1997;29:132-5.

23. Haas M, Rajaraman S, Ahuja T, Kittaka M, Cavallo T. Fibrillary immunotactoid glomerulonephritis in HIVpositive patients: A report of three cases. Nephrol Dia Transplant. 2000;15:1679-83. 